\title{
Research on elastic modulus measurement system based on STM32
}

\author{
Feng Liu
}

\author{
Wuhan Textile University, Hubei, China
}

Keywords:elastic modulus;embedded;STM32;deformation; measure.

\begin{abstract}
Elastic modulus, which can be used as the the difficulty of material's producing elastic deformation is an important parameter in the engineering application.At present, the method of measuring modulus of elasticity is the original manual measurement.In this text,i want to talk about one method using an embedded processor to measure the elastic modulus automatically. The system uses STM32 as the core, generates a horizontal direction force by controlling the motor, with a tension pressure sensor ${ }^{[1]}$ to measure the tension magnitude, and then the micro deformation of the object under test is obtained by laser ranging. At last,we calculate the elastic modulus by Hooke's law.
\end{abstract}

\section{Introduction}

Elastic modulus is an important parameter of engineering materials. When it's larger, the required stress for a certain deformation of the material is stronger,the material stiffness is bigger. So under the action of a certain stress, material changes in the elastic deformation of the elastic modulus is smaller. The elastic modulus $\mathrm{E}$ is the stress required to produce a unit elastic deformation under applied stress, it is an index that reflects the material's ability to resist elastic deformation. According to Hooke's law and the principle of the combination of embedded system, automatic measurement system of elastic modulus in the study design, in order to achieve and improve the measurement speed and reduce the error of manual access measurement.

\section{Elastic Modulus And Measurement}

\subsection{Definition Of Elastic Modulus}

The material in the elastic deformation stage, The stress is proportional to deformation, The coefficient of proportionality is called the modulus of elasticity ${ }^{[2]}$, its unit is Delta per square centimeter.

\subsection{Experimental Measurement Principle}

Measurement of elastic modulus by tensile method: A steel wire with a length of $\mathrm{L}$ and a cross sectional area of $\mathrm{S}$ is set. It is deformed under the action of external force $\mathrm{F}$, and is obtained by Hooke's law:

$$
\frac{F}{S}=Y \frac{\Delta L}{L}
$$

In this equation $\Delta L$ is the shape variables, $\mathrm{Y}$ is the elastic modulus, the unit is N.M-2

assuming that the material diameter is $\mathrm{D}$, then $\mathrm{S}=0.25 \pi \mathrm{D} 2$, Bringing this equation to the upper equation can be obtained:

$$
Y=\frac{4 F L}{\pi D^{2} \Delta L}
$$

It can be seen that when the material under test is certain, the $\mathrm{L}$ and $\mathrm{D}$ are known, and the elastic modulus of the material being measured can be measured as long as the deformation and stress are accurately measured.

\section{System Design Scheme}

The main work of the system is to accurately measure the deformation of materials under stress, Therefore, in the design of the system, STM32 is used as processor ${ }^{[3]}$, to control the motor to pull material to deformation, tension was measured by using high precision force sensor, because of extremely small deformation is not easy to distinguish, by way of laser ranging error is small. The overall system diagram is shown in Figure 1.

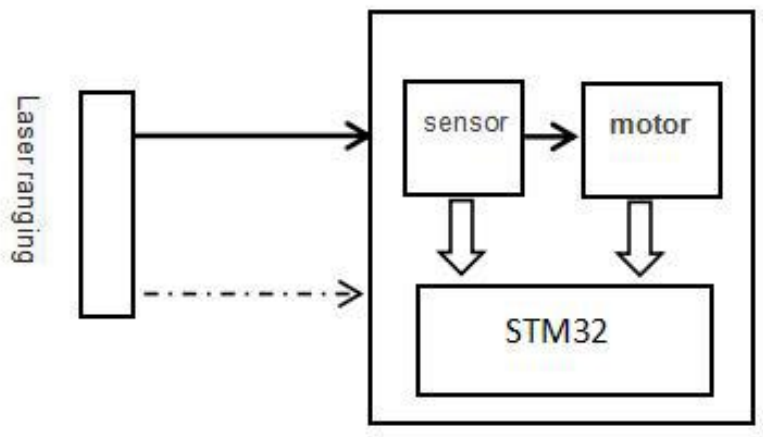

Figure 1. The overall system diagram

\subsection{Measurement Of Deformation}

When the system controls the rotation of the motor, that is, the stretching material, when the shape variable $\Delta L$ is very small, the use of general methods of measurement, prone to errors, and poor consistency, so the system uses laser ranging methods, motor movement before measuring the current length of L1, the length of deformation after measuring $\mathrm{L} 2, \Delta L=L 2-L 1$. The choice of parameters for laser ranging 
is shown in table 1 .

\begin{tabular}{|l|l|l|l|}
\hline $\begin{array}{l}\text { Measuring } \\
\text { Range }\end{array}$ & $\begin{array}{l}\text { Measurement } \\
\text { Accuracy }\end{array}$ & Power & $\begin{array}{l}\text { Communication } \\
\text { Mode }\end{array}$ \\
\hline $0.02-100 \mathrm{~m}$ & $\pm 2 \mathrm{~mm}$ & $<1 \mathrm{mw}$ & serial port \\
\hline
\end{tabular}

Table 1. Parameters Selection of laser ranging

\subsection{Stress Measurement}

In the measurement of stress, due to the high accuracy, the system adopts high precision S pressure sensor, use of alloy steel with high fatigue strength and good toughness can be long time stress, the internal use of temperature compensation resistance, reduce the temperature of the output signal of the perturbation. The performance parameters such as table 2 .

\begin{tabular}{|c|c|c|}
\hline Sensitivity & $\begin{array}{c}\text { Nonlinear } \\
(\% \mathrm{~F} . \mathrm{S})\end{array}$ & $\begin{array}{c}\text { Repeatability } \\
(\% \mathrm{~F} . \mathrm{S})\end{array}$ \\
\hline $2 \pm 0.05 \mathrm{mv} / \mathrm{V}$ & \pm 0.03 & \pm 0.03 \\
\hline
\end{tabular}

Table 2. Sensor Parameters

\subsection{Pulling Force Selection}

The tension of the system is controlled by the STM32 motor to generate tension, because of the accuracy of the system requirements in the $\mathrm{mm}$ level, so the system can be used to complete the electric cylinder. Electric cylinder parameters such as table 3 .

\begin{tabular}{|c|c|c|c|}
\hline Trip & $\begin{array}{c}\text { Push-pull } \\
\text { effort }\end{array}$ & $\begin{array}{c}\text { Repeat positioning } \\
\text { accuracy }\end{array}$ & Pitch \\
\hline $100 \mathrm{~mm}$ & $40 \mathrm{~kg}$ & $0.02 \mathrm{~mm}$ & 2.5 \\
\hline \multicolumn{3}{|c|}{ Table 3. Parameters Of Electric Cylinder }
\end{tabular}

\subsection{System Architecture}

The deformation of the system to accurately measure the measured materials, and materials in stress under the weak deformation, therefore need to take into account the deformation problem of other devices, such as the deformation $^{[4]}$ of the sensor, in this system, we use the reasonable layout to eliminate part of the error sensor for deformation under stress.

As shown in figure 1, the most left in the system, we set up the fixed end, determine the position of the laser sensor at the same time, in the middle of the left and right system set to a fixed position of the tested material, at the same time provide measurement point for laser ranging.Join to pull on the right side of the measurement point pressure sensor, the deformation of the measured material only associated with the deformation of material, deformation of the sensor are excluded outside the system.

\section{System Software Design}

\subsection{Software Flow Chart}

In the system, STM32 is used as a processor, which is rich in resources, there are five serial communication ports, with $\mathrm{AD}$ conversion function, at the same time there are a large number of IO can be used in this system for laser ranging by serial communication, the sensor output analog signal measurement, so we use AD conversion function, the display part LCD display.The main flow chart of software are shown in Figure 2.

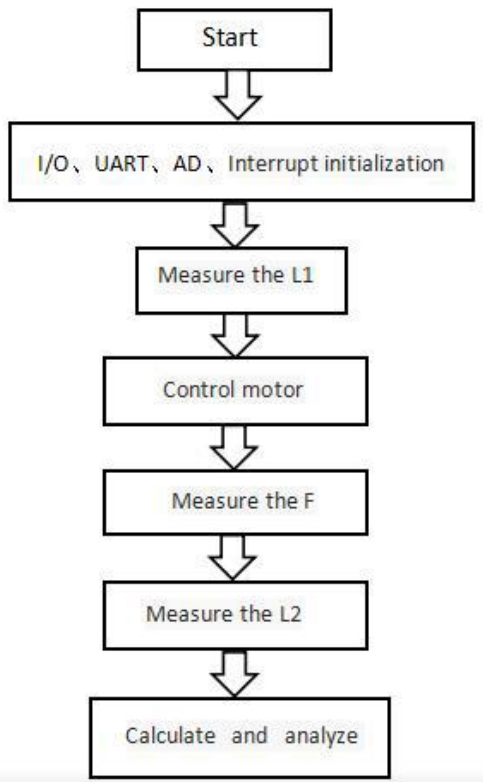

Figure 2. The main flow chart of software

\subsection{Software Design Of Analog To Digital Conversion}

STM32 has three independent digital analog conversion module, the conversion precision of 12 , because the system only needs to sample a AD signal, so using ADC1. as the clock configuration for the $72 \mathrm{MHz}$, so $\mathrm{AD} t=1.17 \mathrm{~s}$. The conversion time of the system to continue sampling voltage value to determine the motor position and stop action therefore, the system requires the use of a single continuous conversion mode, in order to improve the efficiency of the system using the transmission mode of DMA processor, AD will mark the end of EOC in every time the end of conversion, the system also generates the DMA request, DMA will convert DR data transmission To the storage unit. Using the DMA method in STM32: after the configuration enabled peripherals DMA event request, then can make the corresponding DMA channel, the target address setting data source address and data transmission, start the conversion can be carried out after transmission.

\subsection{Simple Data Processing Algorithm}

Make $Y \frac{S}{L}=\frac{F}{\Delta L}$ simple transformations , the material is certain, $\mathrm{S}$ and $\mathrm{L}$ are known, let $S / L=K$, so we may draw $Y \bullet K=\frac{F}{\Delta L}$

L2.From this we can see that the data model is a meta data model, and the least square method can be used to process the data and get the optimal value of the data. 


\section{Conclusion}

Using the principle of elastic modulus measurement ${ }^{[5]}$, and Hooker's law,meta data model.If measure out of shape variables $\Delta L$ and the stress $F$ can be used to measure the modulus of elasticity of materials. This paper uses STM32 as the core processor, the accurate measurement of materials using laser ranging mode shape variable, using stress pull pressure sensor measuring material, and then through reasonable system structure, reduce the system error, the data were processed by the least squares method, get the optimal value.

In this system, a method of measuring elastic modulus with embedded system is studied, which reduces the error caused by manual participation. The measurement accuracy is high, the speed is fast, and the error is small.

\section{References}

[1] Zi Xinyun, Zhao Shufan, Geng Shuai. Strain type torque sensor analysis and ANSYS simulation [J]. Instrument technology, 2014 (10): 50-54.

[2] Xie Heping, Dong Yuli. The elastic modulus method in the definition of classical damage $[\mathrm{J}]$. mechanics and practice, 1997,19 (2): 1-5.

[3] Yuan Yadi. Design and implementation of smart home system based on Internet of things [J]. enterprise technology development monthly, 2015,34 (21): 8-8.

[4] Tu Ping. Statistical analysis of sensor linearity [J]. sensor technology, 2000,19 (3): 36-37.

[5] Zhang Jianren, Wang Haichen, Yang Weijun. Experimental study on early compressive strength and modulus of elasticity [J]. Chinese and foreign highway, 2003,23 (3): 89-92. 